\title{
Left internal mammary spasm mimicking graft dissection in the course of percutaneous coronary intervention of anastomotic in-stent restenosis
}

\author{
Maciej T. Wybraniec ${ }^{1,2}$, Andrzej Kubicius ${ }^{2,3}$, Katarzyna Mizia-Stec ${ }^{1,2}$ \\ ${ }^{1}$ First Department of Cardiology, School of Medicine in Katowice, Medical University of Silesia, \\ Upper Silesia Medical Center, Katowice, Poland \\ ${ }^{2}$ Upper Silesia Medical Center, Katowice, Poland \\ ${ }^{3}$ Department of Cardiology in Cieszyn, Upper Silesia Medical Center, Katowice, Poland
}

The percutaneous coronary intervention (PCI) of left internal mammary artery (LIMA) bypass to left anterior descending artery (LAD) confers high risk of intractable spasm and graft dissection. This case presents a 67-year-old male, following coronary artery bypass grafting and PCI of LIMA-LAD anastomosis with drug-eluting stent implantation 7 months prior to index hospitalization, who currently presented with inferior wall ST-segment elevation acute myocardial infarction. The coronary angiography performed via right femoral approach showed new significant $90 \%$ stenosis within the first diagonal branch and $99 \%$ in-stent restenosis in LIMA-LAD anastomosis (Fig. 1A). First, the lesion in $\mathrm{LAD} /$ diagonal branch was predilated with a $2.5 \mathrm{~mm}$ balloon and a $2.5 \times 25 \mathrm{~mm}$ sirolimus-eluting stent was implanted. Second, an internal mammary artery guiding catheter was employed and the Whisper $\mathrm{LS}^{\circledR}$ guidewire was advanced across the LIMA-LAD restenosis. The lesion was initially predilated with the $2.5 \mathrm{~mm}$ balloon and a $2.0 \times 23 \mathrm{~mm}$ everolimus-eluting stent was implanted (Fig. 1B). Following stent deployment, a severe impairment of LIMA-LAD flow was documented (Fig. 1C, Suppl. Movie 1), which was accompanied by aggravation of restrosternal chest pain and reduction of blood pressure (90/60 $\mathrm{mmHg})$. Although LIMA dissection was suspected, double intracoronary bolus of diluted $0.1 \mathrm{mg}$ nitroglycerine led to gradual restoration of Thrombolysis in Myocardial Infarction 3 blood flow (Fig. 1D) and, paradoxically, improvement of hemodynamic status $(130 / 80 \mathrm{mmHg})$. The patient was discharged home following an uneventful further in-hospital stay. Iatrogenic periprocedural LIMA constriction should always be suspected and vasodilative agents utilized in cases of impaired flow during LIMA PCI.

Conflict of interest: None declared

Address for correspondence: Maciej T. Wybraniec, MD, PhD, First Department of Cardiology, School of Medicine in Katowice, Medical University of Silesia, ul. Ziołowa 47, 40-635 Katowice, Poland, tel: +48 3235988 90, fax: +48 3225230 32, e-mail: maciejwybraniec@gmail.com 

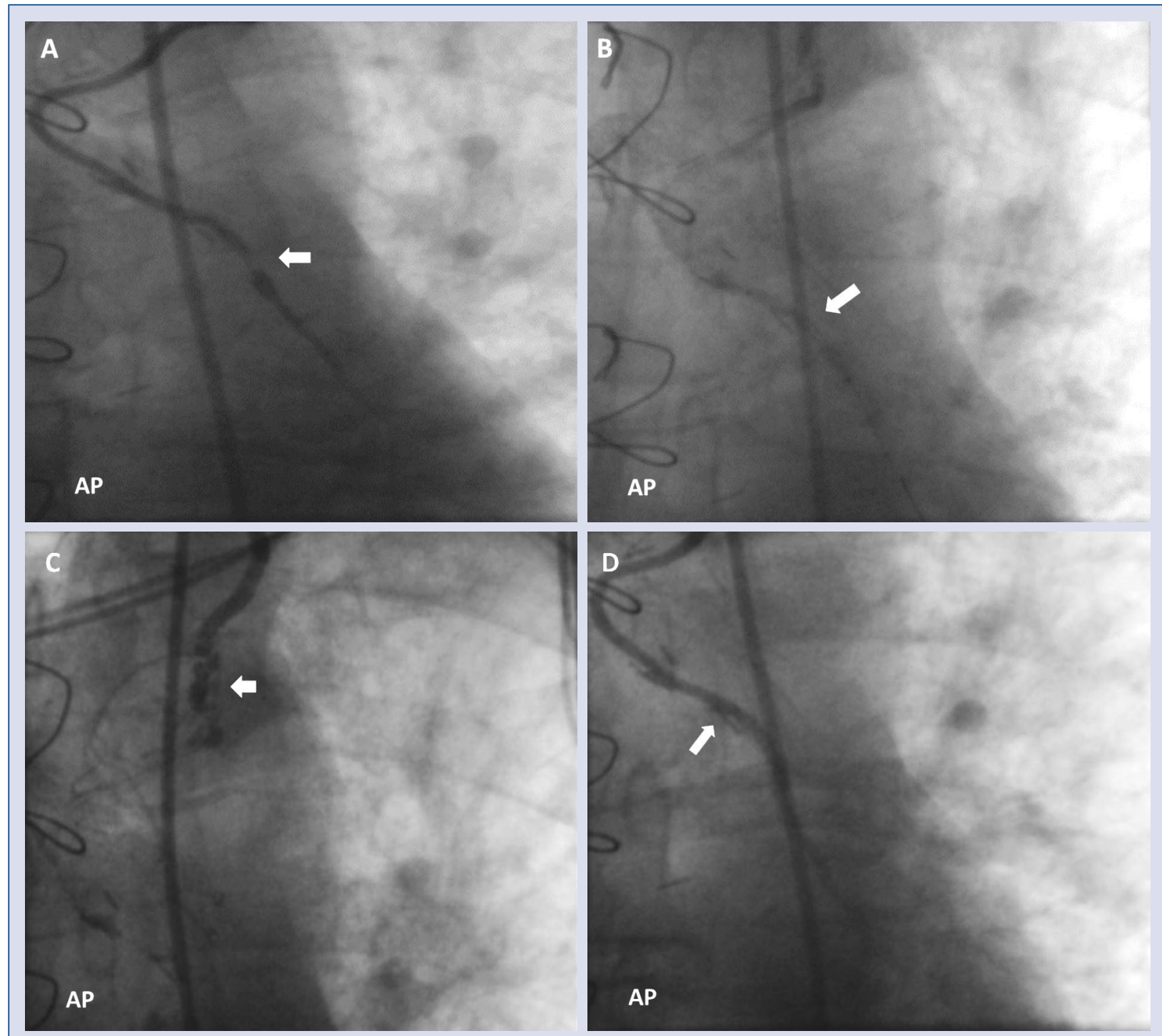

\section{AP}

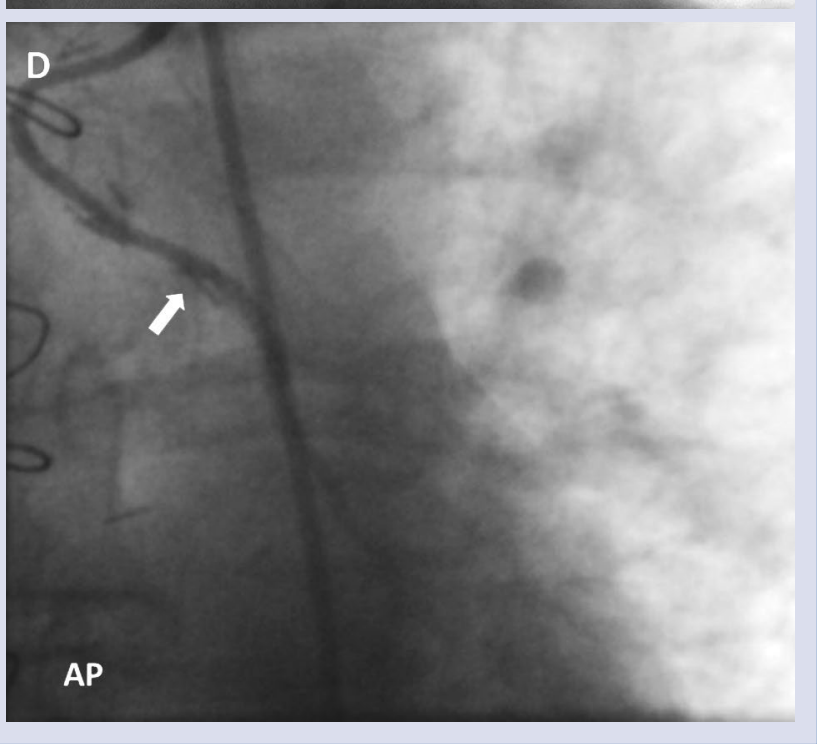

Figure 1. Coronary angiography performed via right femoral approach. A. Anterior-posterior (AP) view, arrow - $99 \%$ in-stent restenosis in left internal mammary artery (LIMA) to left anterior descending artery anastomosis; B. AP view; arrow - implantation of evrolimus-eluting stent within the anastomotic in-stent restenosis; C. AP view; arrow dissection-like impairment of flow within the arterial graft due to severe spasm of LIMA; D. AP view; arrow - restoration of Thrombolysis in Myocardial Infarction grade 3 blood flow after intracoronary administration of nitroglycerine. 